\title{
Cardiovascular nuclear magnetic resonance: basic and clinical applications
}

\author{
John R. Forder and Gerald M. Pohost \\ Division of Cardiovascular Medicine, Keck School of Medicine, The University of Southern California, Los Angeles, California, USA \\ J. Clin. Invest. 111:1630-1639 (2003). doi:10.1172/JCI200318868.
}

\section{Brief history}

Felix Bloch (1) at Stanford University and Edward Purcell and his colleagues (2) at Harvard University reported the phenomenon of NMR independently in 1946. As a result, Bloch and Purcell shared the 1952 Nobel Prize in Physics. Between 1950 and 1970, NMR spectroscopy was developed and used to analyze chemical and physical molecular structure. In 1971, Raymond Damadian reported that the NMR relaxation times of tumors differed from those of normal tissue, suggesting for the first time that magnetic resonance (MR) might be used for the detection of disease (3). In 1973, Paul Lauterbur was the first to report that images could be generated by NMR using small test tube samples of water and oil (4). Rather than creating a homogeneous magnet field by adjusting the "shimming" magnets to minimize field inhomogeneity, Lauterbur applied a magnetic field gradient to induce inhomogeneity in a planned way, providing a method to encode different parts of the substance to be imaged. He generated images using a technique analogous to that employed in $\mathrm{x}$-ray computed tomography, known as back-projection-reconstruction. Two years later, Richard Ernst and colleagues proposed using the mathematical operation of Fourier transformation to create a spatial image from the frequencies generated by radiowave excitation within a magnetic field (5). It was an additional five years before Ernst and colleagues' ideas were applied, and they quickly formed

\footnotetext{
Address correspondence to: Gerald M. Pohost, Division of Cardiovascular Medicine, Keck School of Medicine, 355 San Pablo Street, no. 117, The University of Southern California, Los Angeles, California 90033, USA. Phone: (323) 442-5480; Fax: (323) 442-5481; E-mail: pohost@usc.edu.

Conflict of interest: The authors have declared that no conflict of interest exists.

Nonstandard abbreviations used: magnetic resonance (MR); echo-planar imaging (EPI); cardiovascular MR (CMR); volume of interest (VOI); chemical shift imaging (CSI); image-selected in vivo spectroscopy (ISIS); spatial response function (SRF); radiofrequency $(\mathrm{RF})$; gadolinium-diethyleneaminepentaacetate (Gd-DTPA); phosphocreatine (PCr); inorganic phosphate (Pi); tricarboxylic acid (TCA); spin-lattice relaxation time (T1); spin-spin relaxation time (T2); change in spin-spin relaxation time due to magnetic field inhomogeniety $(\mathrm{T} 2 *)$.
}

the basis of most modern imaging techniques. Supposedly to avoid confusion with nuclear medicine, the clinical NMR imaging tool became known as magnetic resonance imaging (MRI) in the late 1970s. In 1977, Damadian and colleagues demonstrated MRI of the whole body (6). In this same year, Peter Mansfield developed the echo-planar imaging (EPI) technique for high-speed imaging (7). Also in that year, both Jacobus and colleagues (8) and Garlick and associates (9) published the first NMR phosphorus spectra from isolated perfused rat hearts, which paved the way for numerous publications on myocardial high-energy phosphate metabolism in the heart. In 1980 Goldman and his colleagues described the potential applications of NMR imaging in the assessment of the cardiovascular system (10). Many of the predictions made in that paper have gradually approached fruition. Despite the considerably more frequent use of cardiovascular MR (CMR) imaging compared with CMR spectroscopy, currently, there is significant clinical information to be ascertained from molecules in the myocardium that can be detected by clinical spectroscopy. This article will describe the state-of-the-art of imaging and spectroscopy and provide a framework for suggesting the future applications of this formidable technology.

\section{NMR spectroscopy and imaging techniques}

There are many approaches available, related to the sequence of radiofrequency pulses and magnetic field gradients, for the generation of clinical images and spectra by NMR. The trend has been toward faster imaging sequences and the reduction of artifacts, while spectroscopy development has concentrated on localization techniques - reduction of the volume of interest (VOI), decreased contamination from areas outside the VOI, and increased speed of acquisition. A comparison and summary of some common clinical imaging sequences is provided in Table 1.

\section{Spectroscopic localization techniques}

The clinical application of cardiac NMR spectroscopy requires localization of the observed signals to the in situ myocardium to avoid contamination from adjacent tissues. Although the bulk of clinical myocardial 
spectroscopy has been applied to the evaluation of the metabolites that contain phosphorus, these techniques are applicable to other NMR-visible nuclei, including hydrogen, carbon-13, fluorine-19, and sodium-23. Several techniques have been developed to isolate the VOI; most use the imaging gradients to define an excitation volume, and then turn off the gradients during the subsequent acquisition. The techniques can be broadly grouped into two categories: single volume (Table 2 ) and multiple volumes. Table 3 provides a comparison of the regions localized in each technique. Single-volume techniques usually generate a cylinder of excitation using the lateral margins of a surface coil. Spatial localization is applied only in the direction perpendicular to the plane of the surface coil, resulting in signal acquisition at distinct distances from the surface coil, and relying on the diameter of the coil to provide localization in the planes parallel to the coil. Multivolume techniques are the most widely used clinically and can interrogate multiple sites within the tissue by the use of several gradients. Chemical shift imaging (CSI) is a prime example of multivolume techniques and is described below.

\section{Chemical shift imaging}

CSI is becoming the method of choice for clinical spectroscopy studies of the brain $(11,12)$. As with any conventional Fourier MRI method, CSI uses pulsed phaseencoding gradients to control the localization of the signals from within the tissue. It has an important advantage in that an image is acquired, i.e., many spectra from a grid across the heart can be reconstructed. Thus, CSI is a very efficient method in terms of sensitivity. By voxel shifting, it is also possible to position the center of the VOI retrospectively at the desired location $(13,14)$. Nevertheless, there are drawbacks with the use of CSI for localization (11,15-17). In contrast to imageselected in vivo spectroscopy (ISIS), in which the shape

Table 1

Comparison of common clinical imaging sequences ${ }^{A}$

Spin echo

Among the first useful pulse sequences to be used in cardiovascular imaging

Consists of an excitation pulse, followed by a $180^{\circ}$ pulse

In cardiac studies, excitation pulses must be synchronized with the cardiac cycle; arrhythmias cause problems

Ventricular cavity appears darker, resulting in "dark blood" images

Advantage: The $180^{\circ}$ pulse refocuses bulk susceptibility effects

Disadvantage: Only one echo is acquired per pulse; therefore, it is slow

Gradient-recalled echo (GRE)

Dephasing spins refocused using gradients

Lower flip angles

Shorter repetition time (TR)

Depicts the blood pool with high signal, resulting in "bright blood" images

Advantage: Imaging speed is fast

Disadvantage: Sensitivity to T2* effects is increased; bulk susceptibility effects are not refocused

Fast spin echo (FSE)

Single radiofrequency excitation

Multiple echoes (by using a train of $180^{\circ}$ refocusing pulses)

Signal-to-noise ratio is increased because more $\mathrm{T} 1$ recovery occurs between excitation pulses

Advantage: Refocuses bulk inhomogeneities; image acquisition is fast

Disadvantage: RF power deposition

Echo-planar imaging (EPI) and multi-shot EPI

Multiple echoes are generated following a single excitation pulse by rapid switching of the gradients

All phase-encoding steps can be acquired in a single acquisition

Multi-shot EPI - where the image is constructed from two or more EPI excitations - is more robust, although some temporal resolution is lost

Advantage: Very fast

Disadvantage: High sensitivity to motion, flow, and local magnetic field inhomogeneities can result in gross distortions of image

Steady-state free precession (SSFP) techniques

Coherent technique that uses a fully balanced gradient waveform in each repetition time to maintain steady-state magnetization

Image contrast determined by $\mathrm{T} 2 * / \mathrm{T} 1$ properties

Speed and motion insensitivity make the technique reliable, even in patients who have difficulty with holding their breath

Ideally suited to cardiac cine MR

High contrast between blood and endocardium

Advantage: Fast

Disadvantage: Sensitive to motion and flow

${ }^{A}$ Assuming identical spatial resolution between sequences. T1, spin-lattice relaxation time; T2*, change in spin-spin relaxation time due to magnetic field inhomogeniety. 
Table 2

Single-volume spectroscopic localization techniques

Depth resolved surface coil spectroscopy (DRESS)

Developed by Bottomley et al. $(107,108)$

Used extensively to study cardiac metabolism in various pathologies, including transplant rejection, myocardial ischemia and infarction, and dilated cardiomyopathy $(47,109,110)$

Provides spectra from a series of contiguous cylindrical slices parallel to the surface coil

Point-resolved spectroscopy (PRESS)

Spectra acquired from a single voxel

Often employed when the metabolites of interest are uncoupled and have relatively long $\mathrm{T} 2$ values

There may be a significant increase in signal-to-noise ratio over other sequences under these conditions

Image-selected in vivo spectroscopy (ISIS)

Most extensively used for clinical protocols, mainly because of availability (111-113)

Only a single sensitive volume can be selected at a time

Sensitive to motion artifacts; localization relies on a difference scheme

Selective inversion pulses are applied along three orthogonal axes to localize a single volume

$\mathrm{VOI}$ can be placed anywhere within the sensitive volume of the coil

Can be used to query multiple volumes, albeit in sequential fashion

Stimulated echo acquisition mode (STEAM)

Excellent for the study of coupled spin systems (such as carbon-carbon interactions that are observed in ${ }^{13} \mathrm{C}$ spectra of glutamate)

Rapid repetition and short acquisition times maximize signal acquisition

Use of stimulated echoes may make quantitation problematic $(114,115)$

Spatial localization with optimal pointspread function (SLOOP)

Often combined with chemical shift imaging (CSI) techniques (see below) to permit more accurate quantitation (116-118)

Defines a point-spread function that weights spectral contribution according to the spatial response function that defines the VOI

A priori knowledge is used regarding anatomic structures that are examined

Signals can be acquired from voxels of any shape

Contamination from outside the volume(s) of interest

T2, spin-spin relaxation time.

of the VOI (at least in theory) closely resembles a cube with steep edges, the shape of the sensitive volume in CSI is given by the spatial response function (SRF). The SRF indicates the weight by which each point in space contributes (in amplitude and phase) to some spectrum. The shape of the SRF in conventional, unfiltered CSI looks like a sinc-function. There is no well-defined transition from within the VOI to outside the VOI, and, as a result, there can be significant contamination from outside the region of interest. CSI is also subject to motion artifacts, because the reconstruction of the local spectra relies on a linear combination of signals that have been collected at different time points throughout the experiment. Whatever localization method is used, the fundamental limitation is dictated by sensitivity. Appropriate coil design, careful positioning of the patient, and optimal adjustment of the measurement method are necessary for successful results. Nevertheless, one will always have to compromise among the signal-to-noise ratio the experiment duration, and either spatial resolution or contamination.

\section{Current imaging applications}

\section{Peripheral angiography}

Among the most widely used MR applications is peripheral angiography. Due to the signal generated by blood motion, the blood vasculature is readily visualized. But angiography had its beginnings using spin labeling. In the 1980s, Singer used labeling of a blood vessel in the leg, for example, and examination of the distal radiofrequency (RF) emission distally to determine vascular blood velocity (18). Now, with the addition of paramagnetic contrast agents, rapid and high-resolution imaging can be performed in the cerebrovasculature, the aorta and the ileofemoral arterial systems, thereby obviating the need for catheter-based angiography.

\section{Myocardial-viability assessment:} delayed contrast enhancement

Following the observations by Judd, Kim, and colleagues (19-22) that areas of infarcted or scarred myocardium accumulate and retain contrast agent 10 or more minutes after administration, delayedenhancement imaging has quickly found utility as a potentially important clinical application for the evaluation of viability in myocardial ischemic syndromes. The technique is relatively straightforward. Gadolinium-diethyleneaminepentaacetate (Gd-DTPA) is administered intravenously, and following a waiting period of 10 or more minutes, imaging is performed using an inversion-recovery pulse sequence. The inversion time is chosen based on the blood concentration of Gd-DTPA (21) in order to reduce myocardial intensity to a low level. With a low level of myocardial intensity, the ability to depict enhancement is improved. Areas of infarc- 
Table 3

Summary of techniques for localizing NMR spectroscopy

\begin{tabular}{lcc}
\hline Application & Technique & Region localized \\
Querying single volumes & DRESS & Single slice \\
& ISIS & Single volume \\
& PRESS & Single volume \\
& STEAM & Single volume \\
Querying multiple volumes & 1D CSI & Multiple slices \\
& 2D CSI & Multiple volumes \\
& within a single slice \\
& 3D CSI & Multiple volumes \\
& & within a VOI
\end{tabular}

tion or scar demonstrate signal enhancement, with clear demarcation of the border of the territory of scar or irreversible damage (Figure 1). Although some controversy remains regarding the optimal dose of Gd-DTPA, and the inversion time for accurate assessment of the nonviable or scar tissue, the value of the information gained warrants further investigation so that this technique may become a routine clinical tool.

\section{Myocardial contractile function}

Regional and global contractile function can be readily assessed using MR methods. Due to its dimensional accuracy, high resolution, and 3D properties, MR images are ideally suited for the assessment of left and right ventricular function (Figure 2). Ejection fraction is the most commonly employed approach to assess left ventricular systolic function. However, other methods, such as radionuclide approaches, are capable of accurate assessment of ejection fraction. The application of MRI to the evaluation of cardiac end-systolic and end-diastolic volumes has great utility in clinical practice, for it permits the accurate, noninvasive, repeatable measurement of ventricular volumes and the calculation of cardiac output, stroke volume, and ejection fraction.

\section{Imaging applications for the near future}

\section{Radiofrequency tagging}

Developed by Elias Zerhouni and colleagues (23), a radiofrequency tag is a region within the imaged tissue where the net magnetization has been altered with carefully designed radio frequency pulses. Each tag is created as a $3 \mathrm{D}$ plane that extends through the tissue, and it is seen as a tag line when imaged in an orthogonal view. Because the tags result from alterations of the magnetization of the tissue itself, the motion of the tags matches the motion of the underlying tissue. Various tag-generation schemes have been invented for creating numerous tags in a short amount of time. The more popular tagging schemes include stacks of parallel lines $(13,24)$, grids $(14,25)$, and radial stripes (26). By tracking material points as a function of time, it is possible to compute the local Lagrangian strain tensor, the description of motion around a given point in the tissue as it traverses through time and space. Accurate determination of the extent to which myocardial tissue is contracting is of paramount importance - perhaps second only to determination of viability. Although the concept of radiofrequency tagging has been around for a decade automated software to analyze the images has only become available in recent years (27-32). With the recent inclusion of tagging sequences on all of the major clinical imaging platforms, these techniques will find greater acceptance within cardiovascular medicine.

\section{Phase tracking}

Using the phase information intrinsic to MRI, it is possible to directly measure the rate of change of strain within the myocardium. An alternative means for the calculation of a local Lagrangian strain tensor to follow tissue deformation is to determine the relative velocity of the myocardial tissue on a pixel-by-pixel basis (33, $34)$. The local gradient of the velocity field at a given spatial location permits the computation of the Eulerian strain rate tensor, which is analogous to calculation of the strain tensor from the displacement gradient (as is done with tagging). In a phase-contrast image, the change in phase of the net magnetization inside each pixel, compared with a reference image, correlates to the velocity of the underlying tissue in the direction that is parallel to the applied magnetic field gradient. This method has an advantage over tagging in that each pixel provides a unique motion measurement, and MR phase-contrast imaging has great potential for reconstructing 3D motion (35). The recent development of displacement encoding with stimulated echoes (DENSE) for high-resolution myocardial-displacement mapping uses small first-order gradient moments for velocity encoding $(36,37)$. In addition, DENSE magnitude images exhibit black-blood contrast that allows for better myocardial definition, and the small encoding gradients result in reduced motion-related artifacts in size and number. Routine clinical use currently awaits the implementation of these sequences onto the major clinical platforms, and the development of widely available analysis software.

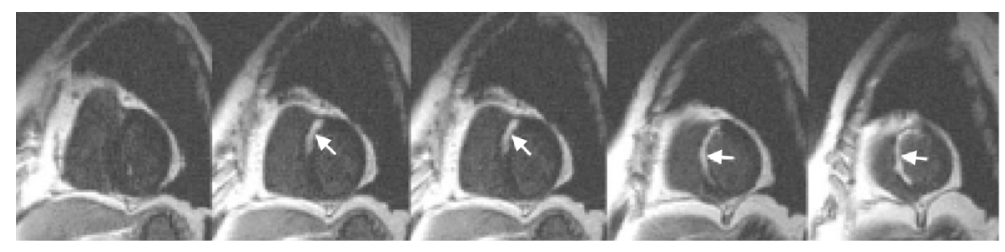

Figure 1

Short-axis views of the heart 32 minutes after contrast injection. The inversion time was adjusted to null the normal myocardium and the area of infarction shows as a bright region. Reproduced with permission from ref. 106. The arrows indicate the territory demonstrating enhancement. 


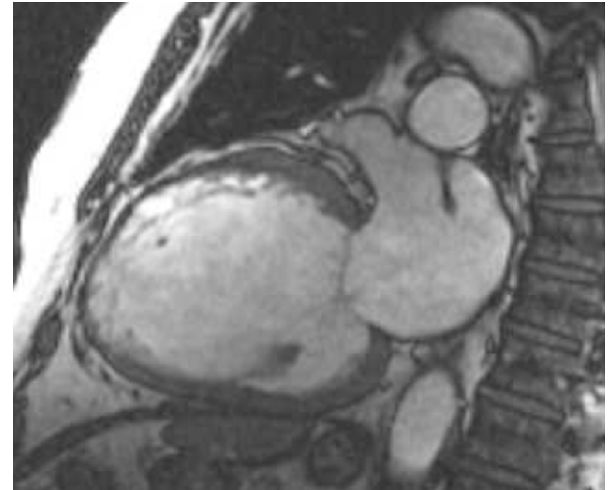

Figure 2

Anatomical detail of the heart is unsurpassed using MRI.

\section{Coronary angiography}

Peripheral angiography (in organs that remain relatively stationary) is quite simple compared with coronary MR angiography (of the moving heart). Nevertheless, the application of MR methods for the imaging of the coronary arteries has progressed at a steady rate over the past decade (Figure 3). The development of higher-speed approaches that acquire images are based on either standard or spiral techniques, each with its advantages and disadvantages. At the present time, clinical application has been useful for the imaging of bypass graft patency and of congenitally anomalous coronary arteries (38-40). Recent work from Kim et al. (41) described an international multicenter trial that demonstrated the present value of coronary artery imaging. The study demonstrated that the left main, the left anterior descending, and the right coronary arteries were well visualized. The circumflex coronary artery was adequately visualized less frequently. While these results are promising, substantial improvement is required to facilitate adequate resolution of secondary branches and stenotic portions of the coronary arteries. With further improvements in imaging speed, this should happen within the next two to three years.

\section{Plaque imaging and characterization}

One of the advantages of MR is its ability to visualize the arteries in cross section. Accordingly, plaque can be seen, its morphology assessed, and its character analyzed. For example, it is possible to determine the constituents of the plaque in the larger noncoronary vessels, e.g., the aorta, the carotids, the iliofemorals, and the renals. While calcium is not visible (creating a region without signal), lipid can be differentiated from fibrosis using differences in relaxation properties. Plaque composition analysis by MR is thought to allow evaluation of plaque stability, and provide a unique clinical approach to assess stability. This may add to the degree of stenosis to predict future occlusive events $(42,43)$. Routine, clinically relevant coronary arterial plaque imaging will require substantial technical development. It is more feasible in the aorta and peripheral arteries.
Image-guided catheter placement

Cinefluoroscopy can be performed using high-speed MR methods. Thus, catheters can be tracked and positioned using MR fluoroscopic real-time imaging. One can envisage a future in which MR rather than x-rays is used to perform catheter-guided cineangiography and perhaps interventions with high resolution, with the potential to characterize plaque and with the ability to image in three dimensions (44).

\section{Myocardial-perfusion imaging}

Contrast agents based on paramagnetism (e.g., gadolinium) or superparamagnetism (e.g., $\mathrm{Fe}^{2+}$ ) can be tracked as they traverse the myocardium after intravenous power injection to assess myocardial perfusion at rest and with a vasodilator (e.g., adenosine). Presently, MR perfusion imaging has been shown to be comparable to radionuclide myocardial-perfusion imaging. One might think that MR would be better for myocardial-perfusion imaging than radionuclides because of its much higher resolution and lack of attenuation (45). However, the presence of paramagnetic contrast in both ventricles can generate artifacts within the interventricular septum that can confound interpretation (46).

\section{Spectroscopic applications for the near future}

${ }^{31}$ Phosphorus stress test

While in relatively low concentrations in the myocardi$\mathrm{um},{ }^{31} \mathrm{P}$ is found in several very important molecules, particularly the high-energy phosphates, ATP, phosphocreatine (PCr), and inorganic phosphates. $\mathrm{PCr}$ is labile, and its concentration rapidly decreases in the presence of a myocardial insult such as ischemia. ATP is considerably more stable and remains intact for prolonged times during ischemic insults. The PCr/ATP ratio has been used as a means of tracking ischemic insults clinically at 1.5 tesla. Several methods, such as 3D CSI and ISIS with low resolution, have been used to generate myocardial spectra at rest and with handgrip stress. With the advent of higher-field (i.e., 3 tesla) and whole-body systems it should be possible to determine the concentration of inorganic phosphates (Pi). The spectral position of the Pi is related to intracellular $\mathrm{pH}$. With a myocardial insult one would anticipate an acidotic environment detectable by the "chemical shift" of the Pi spectral peak. Clinical application was reported originally by Weiss et al. (47), and later by Yabe et al. (48), for detection of significant reduction in the PCr/ATP with handgrip exercise in patients with ischemia consequent to disease of the left anterior descending coronary artery. Recently, Buchthal et al. demonstrated that approximately $20 \%$ of women with chest pain syndromes, but no significant coronary artery disease by $\mathrm{x}$ ray coronary angiography, also had significant reductions in PCr/ATP as part of the NHLBI supported Women's Ischemia Syndrome Evaluation (WISE) study (49). These investigators attributed this to microvascu- 
lar disease. With the chemical shift in $\mathrm{Pi}$, one should be able to detect concomitant acidosis to further corroborate an ischemic basis for this observation. At present, only the anterior wall of the left ventricle can be adequately interrogated, and therefore it ${ }^{31} \mathrm{P}$ myocardial spectroscopy can only be clinically applied to diffuse or to (anterior-wall) left anterior descending coronary artery disease. With the move to higher fields for clinical scanners, novel coil developments, and pulse sequences that can generate a uniform excitation volume, we will ultimately be able to examine the entire left ventricle using ${ }^{31} \mathrm{P}-\mathrm{NMR}$ spectroscopy.

\section{Sodium-23 measurement of sarcolemmal integrity}

Sodium-23 is NMR-sensitive, and imaging of the myocardium in territories with inadequate cell membrane function and sodium accumulation can be detected. Ideally, a safe shift reagent would be developed to allow ready differentiation between intra- and extracellular sodium. No shift agents (which are usually based on paramagnetic dysprosium) are safer for clinical application at this time. We anticipate the development of such agents in the next few years, which would make sodium imaging an ideal clinical tool.

Other techniques with the potential to distinguish intra- from extracellular sodium use quantum filtering (50-54). These sequences separate resonances on the basis of their motion, taking advantage of the higher degree of restriction found in the intracellular environment. To date, cardiovascular application of these techniques has been limited to studies in the isolated perfused heart (55-59), although the skeletal muscles of patients with myotonia dystrophy have been studied (60). Due to the careful set-up required of these pulse sequences, these techniques have not yet found their way into routine clinical practice, but this is an area that might provide the functionality of sodium imaging without the toxicity of the currently available shift reagents.

An additional approach has been to image the sodium content directly, without regard for compartmentalization. The rationale is that loss of ionic homeostasis, tissue edema, or cell lysis will result in an increase in the sodium concentration of any given volume of myocardium (61-67).

\section{Myocardial intracellular lipid content}

With a myocardial ischemic insult, myocardial metabolism shifts from being aerobic (using primarily fatty acids) to being anaerobic (using glycolytic pathways). This results in the accumulation of myocardial lipids that can be visualized by hydrogen (proton) imaging $(68,69)$. Such lipids are usually amphiphilic and can produce deleterious effects on myocardial function and conduction. Using hydrogen spectroscopic imaging, Reeves and colleagues were able to depict the distribution of lipids in the interventricular septum after a myocardial ischemic insult (69). Lipids in the anterior and posterior left ventricular walls were difficult to image because of adjacent adipose tissue that surrounds the myocardium. With higher-resolution imaging strategies, it might be possible to detect lipid in nonseptal myocardium to assess the extent and severity of the ischemic insult.

\section{Imaging applications still several years away \\ Diffusion imaging for myofiber orientation}

Motion artifacts need to be overcome to make diffusion myocardial imaging a useful clinical application, but at the moment, no other technique yields such exquisite anatomical ultrastructural detail. This is because the diffusion of water in the myocardium follows the muscle fibers (70-73), so that orientation of the fibers can be visualized though the use of diffusion tensor MR imaging (DTMRI). Recently, we have reported multiexponential diffusion behavior in heart tissue and have suggested that the diffusion characteristics of the intracellular environment are different from those of the extracellular environment $(74,75)$. If this is correct diffusion imaging may also provide the means to detect myocardial edema, or cell swelling that is associated with the loss of ionic homeostasis prior to cell death (76). The difficulty that remains to be overcome before this will be a useful technique for CMR is that the bulk motion normally associated with cardiac motion is much larger than the diffusion distances to which these sequences are sensitive $(6-9 \mu \mathrm{m})$. Work by Wedeen and colleagues has made significant progress toward the successful application of diffusion tensor imaging to studies of the heart (77-80), but better image-registration and gating strategies need to be implemented before these techniques are clinically useful for the study of myocardial structure and viability. Intelligently targeted contrast agents

One approach currently under investigation $(81,82)$ is the use of very small lipid spheres $(100-250 \mathrm{~nm}$ diameter), usually with a fluorochemical core, that have two main components: a targeting molecule $-\mathrm{a}$

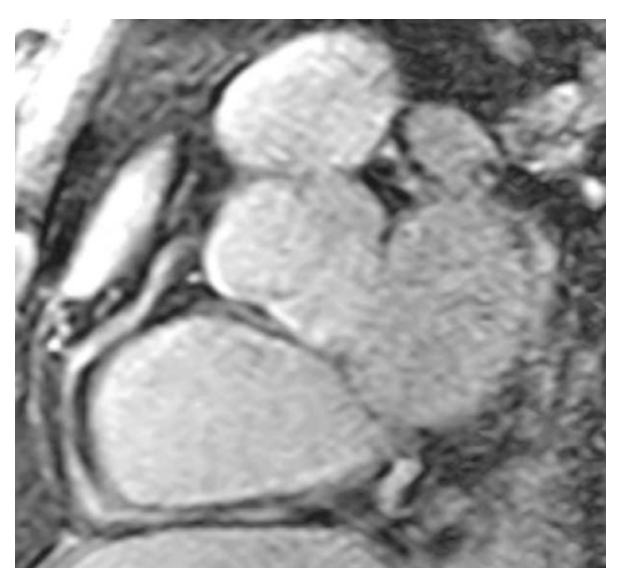

Figure 3

MRI of the right main coronary artery. 
fragment of an antibody, a small peptide, or a ligand for a receptor - used to direct delivery of the particle; and a contrast agent, usually present in high local concentration. Such targeted molecular approaches promise to revolutionize not only disease detection, but also delivery (and monitoring) of site-specific therapeutic agents $(83,84)$. Labeling of either stem cells or macrophages with superparamagnetic iron oxide particles has the potential to track individual cells; however, reduction in image intensity in the presence of superparamagnetic labeled cells could limit their eventual clinical applicability.

\section{Assessing regional oxygen content}

Fluorocarbon blood substitutes. Naturally abundant fluorine $\left({ }^{19} \mathrm{~F}\right)$ exhibits a chemical shift that depends on whether the blood substitute is oxygenated; this shift provides the means for determining the percent oxygenation. Together with the perfluorochemical composition within the core of the targeted contrast-agent nanoparticles mentioned above, this may provide a way to determine regional oxygen content using targeting of cell-specific markers $(85,86)$.

${ }^{17}$ Oxygen imaging and spectroscopy. ${ }^{17} \mathrm{O}_{2}$ is detectable by $\mathrm{MR}$, although the signal-to-noise ratio suffers from poor sensitivity. However, in addition to being directly detectable, oxygen-17 is also paramagnetic, meaning that when the oxygen is reduced to water $\left(\mathrm{H}_{2}{ }^{17} \mathrm{O}\right)$ by mitochondrial electron transport to generate ATP, the protons that are covalently bonded to the ${ }^{17} \mathrm{O}$ result in broadening of the spectral peaks. The amount of broadening is directly proportional to the amount of oxygen that is reduced - and therefore serves as an independent measure of oxygen consumption and resultant ATP production. Although the majority of this work has been in neuroimaging (87-92), this technique may be applied to the heart as well.

Susceptibility imaging. Blood oxygen level-dependent (BOLD) imaging uses the same principles that are used in functional neuroimaging. The ferrous iron in hemoglobin becomes diamagnetic when oxygen is attached but becomes paramagnetic when it is deoxygenated. Determination of the regional oxygen consumption requires measurement of the tissue perfusion and the quantitation of the deoxyhemoglobin content. While BOLD imaging is not yet ready for clinical application to the heart, significant progress is being made (93-98).

\section{Spectroscopic applications still several years away}

Carbon-13 MR spectroscopy

Although NMR's sensitivity to the carbon-13 $\left({ }^{13} \mathrm{C}\right)$ nucleus is considerably lower than its sensitivity to ${ }^{1} \mathrm{H}$ and lower than its sensitivity to ${ }^{31} \mathrm{P},{ }^{13} \mathrm{C}$ could provide insight into some of the most important biochemical pathways, including glycolysis and the tricarboxylic acid (TCA) cycle. At the present time, the relatively low sensitivity has relegated the role of ${ }^{13} \mathrm{C}$ spectroscopy to laboratory investigations. With the commercial transition of clinical systems to higher magnetic fields, and the use of novel pulse sequences designed to improve the sensitivity for detection of ${ }^{13} \mathrm{C}$-enhanced compounds, this nucleus could ultimately find clinical application.

Cross-polarization ${ }^{1} \mathrm{H}\left[{ }^{13} \mathrm{C}\right]-\mathrm{NMR}$ spectroscopy, heteronuclear multiple-quantum coherence (HMQC) spectroscopy, and heteronuclear chemical shift correlation (HETCOR) are strategies for improving the sensitivity with which the ${ }^{13} \mathrm{C}$ nucleus can be detected. They rely on the transfer of magnetization from the carbon to the proton, which improves the signal-tonoise ratio by a factor of approximately eight.

\section{TCA cycle kinetics}

Because the sensitivity of carbon is relatively low, detection of chemicals is limited to those present in concentrations of $100 \mu \mathrm{M}$ or greater. As a result, the concentrations of the intermediates in the TCA cycle are too low for reliable detection. However, $\alpha$-ketoglutarate is in rapid exchange with the intracellular glutamate pool. In myocardium, the intracellular glutamate is present in concentrations ranging from 1.5 to $4 \mathrm{mM}-$ within the range detectable by MR. Estimates of TCA cycle turnover can be made from the incorporation of ${ }^{13} \mathrm{C}$ from energy substrates (such as glucose, or FFAs) into the glutamate pool. By virtue of the role of the TCA cycle the primary means for energy-laden substrate, incorporation rates of ${ }^{13} \mathrm{C}$ from energy substrates into glutamate serve as an indirect estimate of oxygen consumption (99-102). As a result, ${ }^{13} \mathrm{C}$ spectroscopy has been reported to be more sensitive than ${ }^{31} \mathrm{P}$ spectroscopy for the detection of myocardial ischemia, albeit in animal models using very high magnetic fields, e.g., 8.4 tesla (103).

\section{The future: a move to higher fields for cardiac MR}

\section{Diagnostic imaging}

In the future, the ability to image morphology, function, coronary arteries, atherosclerotic plaque, perfusion, metabolic ischemia, and viability potentially within a 60- to 90-minute time interval will make cardiovascular MR the most cost effective and comprehensive means to detect ischemic heart disease and to determine the effect of therapeutic agents. The addition of molecular imaging with NMR will further improve development and assessment of new therapeutic modalities and mechanisms of action. The ability to visualize and track catheters could lead to MR replacing $\mathrm{x}$-rays in the future for improved diagnosis and intervention. The improved signal-to-noise ratio achieved at higher fields can be used to increase imaging speed, and/or to increase spatial resolution. The improvement in spatial resolution comes at the cost of reduction in imaging speed. Thus, the specific goals of a given NMR study must be determined in order to use the improved signal most effectively. The improved spatial resolution in cardiac studies will be of substantial value in perfusion and viability imaging, and spectroscopy. 
Increased imaging speed will reduce motion and flow artifacts, virtually allowing real-time acquisition and display. Analysis of wall-motion abnormalities can be completed in a single breath-hold with excellent signal-tonoise ratio. Due to the increased spin-lattice relaxation times (T1), there is increased RF tag-line persistence through the cardiac cycle at higher fields, simplifying the analysis of regional wall motion by ensuring that the tags continue throughout the cardiac cycle.

Measurement of myocardial tissue perfusion can be classified into two broad categories: those that require administration of an exogenous contrast agent, and those that do not, e.g., spin labeling for measurements of tissue perfusion. Higher magnetic field should result in a greater dynamic range in the myocardial delayed contrast enhancement and, combined with reduced scan times for the fast imaging sequences, greater temporal resolution in quantitating the increase in tissue intensity. Noninvasive perfusion will benefit from the longer $\mathrm{T} 1 \mathrm{~s}$, and the increased signal-to-noise ratio $(95,104)$.

\section{Spectroscopy}

Signal-to-noise ratio increases at higher field, which means that signals that could not be observed at 1.5 tesla can be reliably detected at 3 tesla. This has important implications for cardiac ${ }^{31} \mathrm{P}-\mathrm{NMR}$ spectroscopy, where routine identification of the intracellular inorganic phosphate peak is impossible at 1.5 tesla. The ratio of inorganic phosphate to $\mathrm{PCr}$ is a more sensitive indicator of myocardial ischemia than PCr/ATP.

Furthermore, the higher field increases our ability to distinguish between adjacent peaks in the NMR spectrum. This improvement in spectral resolution means that peaks that overlap at 1.5 tesla may be distinguished at the higher field. This would improve the ability to separate the 2,3-diphosphoglycerate in red blood cells from myocardial inorganic phosphate, for example. Such improved ability to define the inorganic phosphate peak should lead to higher resolution in the measurement of intracellular $\mathrm{pH}$ (calculated from the shift in frequency of the inorganic phosphate peak relative to $\mathrm{PCr}$ ).

Increased field strength also leads to stronger coupling between nuclei. Techniques that improve the detection of relatively weak nuclei, such as ${ }^{13} \mathrm{C}$, will benefit from the shift to the higher field. One technique uses cross-polarization transfer, which transfers the signal from the ${ }^{13} \mathrm{C}$ nuclei to adjacent protons for detection. Higher field increases the signal-to-noise ratio of the proton nuclei directly, but in addition, the coupling between the proton and the carbon is also increased at higher field, resulting in further benefit.

As with imaging, the increased signal-to-noise ratio can be used to increase the speed with which spectroscopic data are acquired, or to decrease the area that is interrogated; and it is of paramount importance in cardiovascular spectroscopic imaging, since issues regarding signal contamination from adjacent tissue (ventricular blood pool, skeletal muscle, or liver) are major concerns.
Traditional spectroscopic localization methods do not use gradients during the data acquisition because of the risk of contamination from signal emanating outside the VOI. This is particularly important with spectroscopic volume selection methods such as ISIS and depth-resolved surface coil spectroscopy (DRESS) that employ gradients to select the region from which spectra are acquired. Gradients are turned off for the data acquisition. However, acquisition speed can be improved by combination of spectroscopic and fast imaging methods. By relaxing of the requirement not to employ gradients during acquisition, rapid imaging strategies such as echo-planar imaging can be incorporated to increase the speed of data acquisition for spectroscopic imaging (105). Recent advances in scanner hardware have made these strategies possible.

\section{Conclusions}

NMR spectroscopy and imaging has been applied increasingly to provide an understanding of the metabolism and function of the heart and blood vessels. The technologies embodied in NMR applications are unique in that they can provide detailed information at molecular, cellular, organ, and whole-body levels, effectively going "from the molecule to man." There has been substantial and remarkable evolution in the cardiovascular application of this technology since the discovery of the phenomenon of NMR in the late 1940s and its application to generate images in the early 1970s. As clinical platforms continue to move to higher fields (and as we overcome the technical hurdles presented by these fields), we obtain new information that has significant impact on the diagnosis and management of our patients. At present, we are still only scratching the surface.

1. Bloch, F. 1946. Nuclear induction. Physical Review (Physics). 70:460-473.

2. Purcell, E., Torrey, H., and Pound, R. 1946. Resonance adsorption by nuclear magnetic moments in a solid. Physical Review (Physics). 69:37-38.

3. Damadian, R.V. 1971. Tumor detection by nuclear magnetic resonance. Science. 171:1151-1153.

4. Lauterbur, P. 1973. Image formation by induced local interactions: examples employing nuclear magnetic resonance. Nature. 242:190-191.

5. Kumar, A., Welti, D., and Ernst, R.R. 1975. NMR Fourier zeugmatography. J. Magn. Reson. 18:69-83.

6. Damadian, R., Goldsmith, M., and Minkoff, L. 1977. NMR in cancer. XVI. FONAR image of the live human body. Physiol. Chem. Phys. 9:97-100.

7. Mansfield, P. 1977. Multi-planar image formation using NMR spin echoes. J. Phys. 10:L55-L58.

8. Jacobus, W.E., Taylor, G.J.T., Hollis, D.P., and Nunnally, R.L. 1977. Phosphorus nuclear magnetic resonance of perfused working rat hearts. Nature. 265:756-758.

9. Garlick, P.B., Radda, G.K., and Seeley, P.J. 1977. Phosphorus NMR studies on perfused heart. Biochem. Biophys. Res. Commun. 74:1256-1262.

10. Goldman, M.R., Pohost, G.M., Ingwall, J.S., and Fossel, E.T. 1980. Nuclear magnetic resonance imaging: potential cardiac applications. Am. J. Cardiol. 46:1278-1283.

11. Pohmann, R., von Kienlin, M., and Haase, A. 1997. Theoretical evaluation and comparison of fast chemical shift imaging methods. J. Magn. Reson. 129:145-160.

12. Kenn, W., Ochs, G., Pabst, T.A., and Hahn, D. 2001. 1H spectroscopy in patients with amyotrophic lateral sclerosis. J. Neuroimaging. 11:293-297.

13. Fischer, M., Bockhorst, K., Hoehn-Berlage, M., Schmitz, B., and Hossmann, K.A. 1995. Imaging of the apparent diffusion coefficient for the evaluation of cerebral metabolic recovery after cardiac arrest. Magn. Reson. Imaging. 13:781-790.

14. Axel, L., and Dougherty, L. 1989. MR imaging of motion with spatial modulation of magnetization. Radiology. 171:841-845. 
15. Neubauer, S., Horn, M., Hahn, D., and Kochsiek, K. 1998. Clinical cardiac magnetic resonance spectroscopy: present state and future directions. Mol. Cell. Biochem. 184:439-443.

16. Schmidt, O., et al. 2000. Artifacts in CSI-measurements caused by the drift of the static magnetic field. MAGMA. 10:167-170.

17. Yabe, T., Mitsunami, K., and Inubushi, T. 1997. Clinical efficacy of threedimensional chemical shift imaging (3D-CSI): a study of 3D-CSI in the human heart and its clinical perspectives for the future. Nippon Rinsho. 55:1839-1843

18. Singer, J.R. 1986. Nuclear magnetic resonance blood flow measurements. Cardiovasc. Intervent. Radiol. 8:251-259.

19. Kim, R.J., Hillenbrand, H.B., and Judd, R.M. 2000. Evaluation of myocardial viability by MRI. Herz. 25:417-430.

20. Judd, R.M., et al. 1995. Physiological basis of myocardial contrast enhancement in fast magnetic resonance images of 2-day-old reperfused canine infarcts. Circulation. 92:1902-1910.

21. Kim, R.J., Chen, E.L., Lima, J.A., and Judd, R.M. 1996. Myocardial GdDTPA kinetics determine MRI contrast enhancement and reflect the extent and severity of myocardial injury after acute reperfused infarction. Circulation. 94:3318-3326.

22. Croisille, P., et al. 1999. Differentiation of viable and nonviable myocardium by the use of three-dimensional tagged MRI in 2-day-old reperfused canine infarcts. Circulation. 99:284-291.

23. Zerhouni, E.A., Parish, D.M., Rogers, W.J., Yang, A., and Shapiro, E.P 1988. Human heart: tagging with MR imaging. A method for noninvasive assessment of myocardial motion. Radiology. 169:59-63.

24. Mosher, T.J., and Smith, M.B. 1990. A DANTE tagging sequence for the evaluation of translational sample motion. Magn. Reson. Med. 15:334-339.

25. Axel, L., and Dougherty, L. 1989. Heart wall motion: improved method of spatial modulation of magnetization for MR imaging. Radiology. 172:349-350.

26. Bolster, B.D., Jr., McVeigh, E.R., and Zerhouni, E.A. 1990. Myocardial tagging in polar coordinates with use of striped tags. Radiology. 177:769-772.

27. Bundy, J.M., and Lorenz, C.H. 1997. TAGASIST: a post-processing and analysis tools package for tagged magnetic resonance imaging. Comput. Med. Imaging Graph. 21:225-232.

28. Young, A.A. 1999. Model tags: direct three-dimensional tracking of heart wall motion from tagged magnetic resonance images. Med. Image Anal. 3:361-372.

29. Ryf, S., Spiegel, M.A., Gerber, M., and Boesiger, P. 2002. Myocardial tagging with 3D-CSPAMM. J. Magn. Reson. Imaging. 16:320-325.

30. Kondo, T., et al. 1992. Evaluation of cardiac motion and function by cine magnetic resonance imaging. Jpn. Circ. J. 56:632-638.

31. Croisille, P., Guttman, M.A., Atalar, E., McVeigh, E.R., and Zerhouni, E.A 1998. Precision of myocardial contour estimation from tagged MR images with a "black-blood" technique. Acad. Radiol. 5:93-100.

32. Bazille, A., Guttman, M.A., McVeigh, E.R., and Zerhouni, E.A. 1994 Impact of semiautomated versus manual image segmentation errors on myocardial strain calculation by magnetic resonance tagging. Invest. Radiol. 29:427-433.

33. Pelc, L.R., et al. 1994. Evaluation of myocardial motion tracking with cine-phase contrast magnetic resonance imaging. Invest. Radiol. 29:1038-1042.

34. Pike, G.B., Meyer, C.H., Brosnan, T.J., and Pelc, N.J. 1994. Magnetic resonance velocity imaging using a fast spiral phase contrast sequence. Magn. Reson. Med. 32:476-483.

35. Drangova, M., Zhu, Y., Bowman, B., and Pelc, N.J. 1998. In vitro verification of myocardial motion tracking from phase-contrast velocity data. Magn. Reson. Imaging. 16:863-870.

36. Aletras, A.H., Ding, S., Balaban, R.S., and Wen, H. 1999. DENSE: displacement encoding with stimulated echoes in cardiac functional MRI. J. Magn. Reson. 137:247-252.

37. Wen, H. 2001. Mapping the velocity vector onto the spin vector: twodimensional velocity-selective spin excitation for MR flow imaging. Magn. Reson. Med. 46:767-772

38. Langerak, S.E., et al. 2002. Detection of vein graft disease using high-resolution magnetic resonance angiography. Circulation. 105:328-333.

39. Langerak, S.E., et al. 2001. Improved MR flow mapping in coronary artery bypass grafts during adenosine-induced stress. Radiology. 218:540-547.

40. Ishida, N., et al. 2001. Mr flow measurement in the internal mammary artery-to-coronary artery bypass graft: comparison with graft stenosis at radiographic angiography. Radiology. 220:441-447.

41. Kim, W.Y., et al. 2001. Coronary magnetic resonance angiography for the detection of coronary stenoses. N. Engl. J. Med. 345:1863-1869.

42. Choudhury, R.P., Fuster, V., Badimon, J.J., Fisher, E.A., and Fayad, Z.A. 2002. MRI and characterization of atherosclerotic plaque: emerging applications and molecular imaging. Arterioscler. Thromb. Vasc. Biol. 22:1065-1074

43. Corti, R., Fuster, V., Badimon, J.J., Hutter, R., and Fayad, Z.A. 2001. New understanding of atherosclerosis (clinically and experimentally) with evolving MRI technology in vivo. Ann. N. Y. Acad. Sci. 947:181-195; discussion 195-198.

44. Spuentrup, E., et al. 2002. Magnetic resonance: guided coronary artery stent placement in a swine model. Circulation. 105:874-879.

45. Zenovich, A., Muehling, O.M., Panse, P.M., Jerosch-Herold, M., and Wilke, N. 2001. Magnetic resonance first-pass perfusion imaging: overview and perspectives. Rays. 26:53-60.

46. Doyle, M., et al. 2003. The impact of myocardial flow reserve on the detection of coronary artery disease by perfusion imaging methods: an NHLBI study. J. Cardiovasc. Magn. Reson. In press.

47. Weiss, R.G., Bottomley, P.A., Hardy, C.J., and Gerstenblith, G. 1990 Regional myocardial metabolism of high-energy phosphates during isometric exercise in patients with coronary artery disease. N. Engl. J. Med. 323:1593-1600.

48. Yabe, T., et al. 1994. Detection of myocardial ischemia by ${ }^{31} \mathrm{P}$ magnetic resonance spectroscopy during handgrip exercise. Circulation. 89:1709-1716.

49. Buchthal, S.D., et al. 2000. Abnormal myocardial phosphorus-31 nuclear magnetic resonance spectroscopy in women with chest pain but normal coronary angiograms. N. Engl. J. Med. 342:829-835.

50. Navon, G., Shinar, H., Eliav, U., and Seo, Y. 2001. Multiquantum filters and order in tissues. NMR Biomed. 14:112-132.

51. Shinar, H., Knubovets, T., Eliav, U., and Navon, G. 1993. Sodium interaction with ordered structures in mammalian red blood cells detected by ${ }^{23} \mathrm{Na}$ double quantum NMR. Biophys. J. 64:1273-1279.

52. Eliav, U., and Navon, G. 1994. Analysis of double-quantum-filtered NMR spectra of $23 \mathrm{Na}$ in biological tissues. J. Magn. Reson. B. 103:19-29.

53. Knubovets, T., Shinar, H., Eliav, U., and Navon, G. 1996. A 23Na multiple-quantum-filtered NMR study of the effect of the cytoskeleton conformation on the anisotropic motion of sodium ions in red blood cells. J. Magn. Reson. B. 110:16-25.

54. Knubovets, T., Shinar, H., and Navon, G. 1998. Quantification of the contribution of extracellular sodium to $23 \mathrm{Na}$ multiple-quantum-filtered NMR spectra of suspensions of human red blood cells. J. Magn. Reson. 131:92-96

55. Schepkin, V.D., et al. 1998. Sodium TQF NMR and intracellular sodium in isolated crystalloid perfused rat heart. Magn. Reson. Med. 39:557-563.

56. Askenasy, N., and Navon, G. 1998. Intracellular volumes and membrane permeability in rat hearts during prolonged hypothermic preservation with St. Thomas and University of Wisconsin solutions. J. Mol. Cell. Cardiol. 30:1329-1339.

57. Askenasy, N., Vivi, A., Tassini, M., and Navon, G. 1996. The relation between cellular sodium, $\mathrm{pH}$ and volumes and the activity of $\mathrm{Na} / \mathrm{H}$ antiport during hypothermic ischemia: multinuclear NMR studies of rat hearts. J. Mol. Cell. Cardiol. 28:589-601.

58. Askenasy, N., Vivi, A., Tassini, M., Navon, G., and Farkas, D.L. 2001. NMR spectroscopic characterization of sarcolemmal permeability during myocardial ischemia and reperfusion. J. Mol. Cell. Cardiol. 33:1421-1433.

59. Navon, G., Werrmann, J.G., Maron, R., and Cohen, S.M. 1994. ${ }^{31}$ P NMR and triple quantum filtered $23 \mathrm{Na}$ NMR studies of the effects of inhibition of $\mathrm{Na}+/ \mathrm{H}+$ exchange on intracellular sodium and $\mathrm{pH}$ in working and ischemic hearts. Magn. Reson. Med. 32:556-564.

60. Kushnir, T., et al. 1997. In vivo ${ }^{23} \mathrm{Na}$ NMR studies of myotonic dystrophy. Magn. Reson. Med. 37:192-196.

61. Jerecic, R., Bock, M., Wacker, C., Bauer, W., and Schad, L.R. 2002. ${ }^{23} \mathrm{Na}$ MRI of the human heart using a 3D radial projection technique. Biomed. Tech. (Berl.) 47(Suppl. 1):458-459.

62. Sandstede, J.J., et al. 2001. Assessment of myocardial infarction in humans with ${ }^{23} \mathrm{Na}$ MR imaging: comparison with cine MR imaging and delayed contrast enhancement. Radiology. 221:222-228.

63. Sikes, P.J., Zhao, P., Maass, D.L., and Horton, J.W. 2001. Time course of myocardial sodium accumulation after burn trauma: a ${ }^{31} \mathrm{P}$ - and ${ }^{23} \mathrm{Na}-$ NMR study. J. Appl. Physiol. 91:2695-2702.

64. Pabst, T., et al. 2001. Optimization of ECG-triggered 3D ${ }^{23} \mathrm{Na}$ MRI of the human heart. Magn. Reson. Med. 45:164-166.

65 . Horn, M., et al. 2001. Detection of myocardial viability based on measurement of sodium content: a ${ }^{23} \mathrm{Na}-\mathrm{NMR}$ study. Magn. Reson. Med. 45:756-764.

66. Kim, R.J., et al. 1999. Relationship of elevated ${ }^{23} \mathrm{Na}$ magnetic resonance image intensity to infarct size after acute reperfused myocardial infarction. Circulation. 100:185-192.

67. Kim, R.J., et al. 1997. Fast ${ }^{23} \mathrm{Na}$ magnetic resonance imaging of acute reperfused myocardial infarction. Potential to assess myocardial viability. Circulation. 95:1877-1885.

68. Evanochko, W.T., Reeves, R.C., Sakai, T.T., Canby, R.C., and Pohost, G.M. 1987. Proton NMR spectroscopy in myocardial ischemic insult. Magn. Reson. Med. 5:23-31.

69. Reeves, R.C., Evanochko, W.T., Canby, R.C., McMillin, J.B., and Pohost, G.M. 1989. Demonstration of increased myocardial lipid with postischemic dysfunction ("myocardial stunning") by proton nuclear mag- 
netic resonance spectroscopy. J. Am. Coll. Cardiol. 13:739-744.

70. Scollan, D.F., Holmes, A., Zhang, J., and Winslow, R.L. 2000. Reconstruction of cardiac ventricular geometry and fiber orientation using magnetic resonance imaging. Ann. Biomed. Eng. 28:934-944.

71. Hsu, E.W., and Henriquez, C.S. 2001. Myocardial fiber orientation mapping using reduced encoding diffusion tensor imaging. J. Cardiovasc. Magn. Reson. 3:339-347.

72. Hsu, E.W., Muzikant, A.L., Matulevicius, S.A., Penland, R.C., and Henriquez, C.S. 1998. Magnetic resonance myocardial fiber-orientation mapping with direct histological correlation. Am. J. Physiol. 274:H1627-H1634.

73. Scollan, D.F., Holmes, A., Winslow, R., and Forder, J. 1998. Histological validation of myocardial microstructure obtained from diffusion tensor magnetic resonance imaging. Am. J. Physiol. 275:H2308-H2318.

74. Forder, J.R., Bui, J.D., Buckley, D.L., and Blackband, S.J. 2001. MR imaging measurement of compartmental water diffusion in perfused heart slices. Am. J. Physiol. Heart Circ. Physiol. 281:H1280-H1285.

75. Hsu, E.W., Buckley, D.L., Bui, J.D., Blackband, S.J., and Forder, J.R. 2001 Two-component diffusion tensor MRI of isolated perfused hearts. Magn Reson. Med. 45:1039-1045.

76. Hsu, E.W., Xue, R., Holmes, A., and Forder, J.R. 1998. Delayed reduction of tissue water diffusion after myocardial ischemia. Am. J. Physiol. 275:H697-H702.

77. Dou, J., Reese, T.G., Tseng, W.Y., and Wedeen, V.J. 2002. Cardiac diffusion MRI without motion effects. Magn. Reson. Med. 48:105-114.

78. Tseng, W.Y., Reese, T.G., Weisskoff, R.M., Brady, T.J., and Wedeen, V.J. 2000. Myocardial fiber shortening in humans: initial results of MR imaging. Radiology. 216:128-139.

79. Tseng, W.Y., Reese, T.G., Weisskoff, R.M., and Wedeen, V.J. 1999. Cardiac diffusion tensor MRI in vivo without strain correction. Magn. Reson. Med. 42:393-403.

80. Reese, T.G., et al. 1995. Imaging myocardial fiber architecture in vivo with magnetic resonance. Magn. Reson. Med. 34:786-791.

81. Flacke, S., et al. 2001. Novel MRI contrast agent for molecular imaging of fibrin: implications for detecting vulnerable plaques. Circulation. 104:1280-1285.

82. Lanza, G.M., et al. 2002. Molecular imaging and targeted drug delivery with a novel, ligand-directed paramagnetic nanoparticle technology. Acad. Radiol. 9(Suppl. 2):S330-S331.

83. Wickline, S.A., and Lanza, G.M. 2002. Molecular imaging, targeted therapeutics, and nanoscience. J. Cell. Biochem. Suppl. 39:90-97.

84. Wickline, S.A., and Lanza, G.M. 2003. Nanotechnology for molecular imaging and targeted therapy. Circulation. 107:1092-1095.

85. Mason, R.P., Jeffrey, F.M., Malloy, C.R., Babcock, E.E., and Antich, P.P 1992. A noninvasive assessment of myocardial oxygen tension: 19F NMR spectroscopy of sequestered perfluorocarbon emulsion. Magn. Reson. Med. 27:310-317.

86. Vink, R. 1992. Nuclear magnetic resonance spectroscopy and the study of tissue oxygen metabolism: a review. Adv. Exp. Med. Biol. 316:187-193.

87. Arai, T., et al. 1991. In vivo oxygen-17 nuclear magnetic resonance for the estimation of cerebral blood flow and oxygen consumption. Biochem. Biophys. Res. Commun. 179:954-961.

88. Pekar, J., et al. 1991. In vivo measurement of cerebral oxygen consumption and blood flow using $17 \mathrm{O}$ magnetic resonance imaging. Magn Reson. Med. 21:313-319.

89. Fiat, D., et al. 1992 . In vivo 17 O NMR study of rat brain during $17 \mathrm{O} 2$ inhalation. Magn. Reson. Med. 24:370-374.

90. Zhu, X.H., Merkle, H., Kwag, J.H., Ugurbil, K., and Chen, W. 2001. 17 O relaxation time and NMR sensitivity of cerebral water and their field dependence. Magn. Reson. Med. 45:543-549.

91. Zhu, X.H., et al. 2002. Development of (17)O NMR approach for fast imaging of cerebral metabolic rate of oxygen in rat brain at high field. Proc. Natl. Acad. Sci. U. S. A. 99:13194-13199.

92. Mateescu, G.D. 2003. Functional oxygen-17 magnetic resonance imaging and localized spectroscopy. Adv. Exp. Med. Biol. 510:213-218.

93. Li, D., Dhawale, P., Rubin, P.J., Haacke, E.M., and Gropler, R.J. 1996. Myocardial signal response to dipyridamole and dobutamine: demonstration of the BOLD effect using a double-echo gradient-echo sequence. Magn. Reson. Med. 36:16-20.

94. Wacker, C.M., et al. 1999. Changes in myocardial oxygenation and perfusion under pharmacological stress with dipyridamole: assessment using T*2 and T1 measurements. Magn. Reson. Med. 41:686-695.
95. Reeder, S.B., Holmes, A.A., McVeigh, E.R., and Forder, J.R. 1999. Simultaneous noninvasive determination of regional myocardial perfusion and oxygen content in rabbits: toward direct measurement of myocardial oxygen consumption at MR imaging. Radiology. 212:739-747.

96. Niemi, P., et al. 1996. Myocardial intensity changes associated with flow stimulation in blood oxygenation sensitive magnetic resonance imaging. Magn. Reson. Med. 36:78-82.

97. Atalay, M.K., Forder, J.R., Chacko, V.P., Kawamoto, S., and Zerhouni, E.A. 1993. Oxygenation in the rabbit myocardium: assessment with susceptibility-dependent MR imaging. Radiology. 189:759-764.

98. Pluim, B.M., et al. 1998. Functional and metabolic evaluation of the athlete's heart by magnetic resonance imaging and dobutamine stress magnetic resonance spectroscopy. Circulation. 97:666-672.

99. Chatham, J.C., Forder, J.R., Glickson, J.D., and Chance, E.M. 1995. Calculation of absolute metabolic flux and the elucidation of the pathways of glutamate labeling in perfused rat heart by 13C NMR spectroscopy and nonlinear least squares analysis. J. Biol. Chem. 270:7999-8008.

100.Weiss, R.G. 1998. 13C-NMR for the study of intermediary metabolism. MAGMA. 6:132.

101. Weiss, R.G., et al. 1992. Indexing tricarboxylic acid cycle flux in intact hearts by carbon-13 nuclear magnetic resonance. Circ. Res. 70:392-408.

102. Malloy, C.R., Sherry, A.D., and Jeffrey, F.M. 1987. Carbon flux through citric acid cycle pathways in perfused heart by ${ }^{13} \mathrm{C}$ NMR spectroscopy. FEBS Lett. 212:58-62.

103.Weiss, R.G., Chacko, V.P., Glickson, J.D., and Gerstenblith, G. 1989. Comparative 13C and 31P NMR assessment of altered metabolism during graded reductions in coronary flow in intact rat hearts. Proc. Natl. Acad. Sci. U. S. A. 86:6426-6430.

104. Reeder, S.B., Atalay, M.K., McVeigh, E.R., Zerhouni, E.A., and Forder, J.R. 1996. Quantitative cardiac perfusion: a noninvasive spin-labeling method that exploits coronary vessel geometry. Radiology. 200:177-184.

105. Pohost, G.M., Meduri, A., Razmi, R.M., Rathi, V.K., and Doyle, M. 2001. Cardiac MR spectroscopy in the new millennium. Rays. 26:93-107.

106. Mahrholdt, H., et al. 2002. Reproducibility of chronic infarct size measurement by contrast-enhanced magnetic resonance imaging. Circulation. 106:2322-2327.

107. Bottomley, P.A. 1985. Noninvasive study of high-energy phosphate metabolism in human heart by depth-resolved 31P NMR spectroscopy. Science. 229:769-772.

108. Bottomley, P.A. 1987. Spatial localization in NMR spectroscopy in vivo. Ann. N. Y. Acad. Sci. 508:333-348.

109.Hardy, C.J., Weiss, R.G., Bottomley, P.A., and Gerstenblith, G. 1991. Altered myocardial high-energy phosphate metabolites in patients with dilated cardiomyopathy. Am. Heart J. 122:795-801.

110. Bottomley, P.A., Weiss, R.G., Hardy, C.J., and Baumgartner, W.A. 1991. Myocardial high-energy phosphate metabolism and allograft rejection in patients with heart transplants. Radiology. 181:67-75.

111.Schaefer, S., et al. 1988. Nuclear magnetic resonance imaging-guided phosphorus-31 spectroscopy of the human heart. J. Am. Coll. Cardiol. 12:1449-1455.

112.Luyten, P.R., Groen, J.P., Vermeulen, J.W., and den Hollander, J.A. 1989. Experimental approaches to image localized human 31P NMR spectroscopy. Magn. Reson. Med. 11:1-21.

113.Twieg, D.B., et al. 1989. Phosphorus-31 magnetic resonance spectroscopy in humans by spectroscopic imaging: localized spectroscopy and metabolite imaging. Magn. Reson. Med. 12:291-305.

114. Moonen, C.T., et al. 1989. Comparison of single-shot localization methods (STEAM and PRESS) for in vivo proton NMR spectroscopy. NMR Biomed. 2:201-208.

115.Turner, R., von Kienlin, M., Moonen, C.T., and van Zijl, P.C. 1990. Single-shot localized echo-planar imaging (STEAM-EPI) at 4.7 tesla. Magn. Reson. Med. 14:401-408.

116.Pohmann, R., Rommel, E., and von Kienlin, M. 1999. Beyond k-space: spectral localization using higher order gradients. J. Magn. Reson. 141:197-206.

117.von Kienlin, M., et al. 2001. Advances in human cardiac 31P-MR spectroscopy: SLOOP and clinical applications. J. Magn. Reson. Imaging. 13:521-527.

118.Loffler, R., Sauter, R., Kolem, H., Haase, A., and von Kienlin, M. 1998. Localized spectroscopy from anatomically matched compartments: improved sensitivity and localization for cardiac 31P MRS in humans. J. Magn. Reson. 134:287-299. 